\title{
A Review of the Medical Weblog, Clinical Correlations
}

\author{
David M. Aronoff, MD and Barbara A. Bartkowiak, MLIS, MST
}

\author{
Keywords: \\ Blogging \\ Internal medicine \\ Internet \\ User-computer interface
}

Corresponding Author:

Barbara A. Bartkowiak, MLIS, MST

Marshfield Clinic

George E. Magnin Medical Library

1000 North Oak Avenue (ML6)

Marshfield, WI 54449

Tel: 7। 5-389-4285

Fax: 7I5-389-5366

Email: bartkowiak.barbara@marshfieldclinic.org

Received: March 16, 2010

Accepted: March 17, 2010

doi: $10.3121 / \mathrm{cmr} .2010 .926$
$\mathrm{W}_{\text {ti }}$ as a way to combine the speed of Internet delivery with more extended discussions either of a personal or professional nature. Because so many of the scientific information sources are behind "pay-walls", 1 it may not be easy to access reliable, substantive literature without affiliation. Even when material is freely available, it may not come in a format that is preferred. Professional-related blogs can fill that void with timely and extended depth of information, and they can build on the known body of knowledge without extensive background repetition for each entry. Basically, blogs can combine factual content, rapidity, and fresh viewpoints and can be tailored to meet users' needs. It is no wonder that 38,000 blogs are created daily. ${ }^{2}$

The evaluation criteria of blogs comprises what one would expect in most electronic media: accuracy of content, bias, credibility of the sources, authority of the writers, attention to grammar and writing acumen, currency and updating of material, tone of contributors, and whether others link to the blog. In the medical arena, credibility of authorship and reliable sources are critical components. While they may be more informal in structure, professional medical blogs require more rigor and substance.

Clinical Correlations (www.clinicalcorrelations.org) is a peer-reviewed internal medicine blog focused on clinical issues. Authors are primarily members of the New York University (NYU) medical community. The content is distributed among 17 specialty categories, including writings on breaking medical research, timely medical headlines, clinical case presentations, and answers to challenging clinical questions. The blog is laid out in a newspaper-like, columnar format. The fonts are all sans serif and easy to read. There is a lead story and links to other sections or categories, such as PrimeCuts: This Week in the Journals. The website features a number of interesting categories, under which can be found some helpful nuggets of information. The site map of the blog provides a broad, general overview of clinical offerings.

One of the authors (D.M.A.), an infectious diseases (ID) specialist, spent a week dipping into Clinical Correlations to sample its general medicine and subspecialty content. What follows is a brief medley of impressions and criticisms of this excellent weblog. A clear-cut strength of this site is the use of peer review. That, coupled with the liberal use of references, provides the kind of evidence-based approach to medicine that is the most helpful to practicing nurses and physicians. This formal, evidence-based approach is counterbalanced by a "bloggish", conversational tone that confers a readability absent in traditional biomedical 
journals. For example, this sample was extracted from a review of the US Food and Drug Administration's decision to approve the use of rosuvastatin for the primary prevention of cardiovascular disease in subsets of healthy adult patients, authored by Dr. Rachel Bond:

As reported by Reuter's in The NY Times Business Section "this clears the way for the drug to be used by millions of people who are not typically prescribed the drug now...likely increasing sales of the drug."[5] This statement leads one to think: does this new approval help in the advancement of preventive medical research for the betterment of our patient population or is this just another way for pharmaceutical companies to make a whopping profit? Hmmm, maybe it's a little bit of both.

The Bedside Rounds section was full of interesting knowledge. One could read about diabetic neuropathy, finger clubbing, and pulsus paradoxus. That section is a bit of a curiosity shop or menagerie of medical topics, but that is to be expected for such a blog category. The Grand Rounds category was particularly enjoyable to peruse. These succinct summaries of lectures given at NYU internal medicine Grand Rounds provided a nice summary of lectures, which most of us could not attend, given by leaders in our field. A feature for the publishers of Clinical Correlations to consider would be to post brief interviews with the physicians delivering the Grand Rounds lectures. Such interviews are often fascinating and provide insight into the motivations and personalities of the dynamic leaders in medicine.

Extra time was spent reviewing ID-related information on Clinical Correlations. There was a potpourri of offerings in ID on the blog, and the majority of the available postings were well-written and interesting. A review on the use of zincbased remedies for the common cold was informative and well-written. It touched on the history of zinc as a cold remedy and reviewed clinical and basic science of the effects of zinc therapies on host defense against common cold viruses. It was pleasing to learn something new through a fascinating tidbit written about zinc-induced anosmia. The fact that ID faculty provided additional evidence-based commentary to supplement primary articles written by house officers and students when needed was also appreciated. A nice example was provided by the interesting article Why is Syphilis Still Sensitive to Penicillin? by Dr. Sam Rougas (with additional commentary provided by Dr. Meagan O'Brien). What is more, at the end of each post, comments can be left by readers. These comments were often more noteworthy than the original story: a major strength. Such "dialogue" between author and reader is an outstanding feature of internet-based learning tools.

A summary of key findings presented at the Infectious Disease Society of America's Annual Meeting was a potentially important contribution to the site. Such a bulleted review could be very helpful for ID providers unable to attend the yearly fall conference. However, the website post reviewing the 2009 meeting (Some gleanings from the meeting of the Infectious Diseases Society of America (IDSA) in Philadelphia, October 29-November 1, 2009) was problematic. Only a brief introduction to the meeting summary was presented on the website, with a link to the full article that simply did not function; linking to the "404: the page you were looking for could not be found" error message was frustrating.

Another problem with this website was the editing. Clinical Correlations uses a peer-review process described on their website, which includes a two-week turn-around time for reviewers and another two weeks for author completion. After acceptance, a senior member of the editorial board reviews the entry, and a copy editor checks the result before publication. One did not need to read very far into most posts to find problems with punctuation or grammar. For example, references were sometimes cited parenthetically, for example "(1)", while in other instances they appeared unguarded, for example " 1 ". In another example, an enzyme (prostaglandin dehydrogenase, PGDH) was abbreviated incorrectly (HPGD). These errors highlight a predictable consequence of the shift in how medical information is disseminated: rapidly, without the usual filters of the trained copy editors who are employed by standard journal publishers. While distracting, such mistakes are often benign. However, if drug doses are written incorrectly or if positive statements (such as, "do this") inadvertently become negative statements (such as "don't do this") then errors might become more consequential. We hope the editing of such weblogs will improve over time.

In summary, Clinical Correlations is a successful blog that will be of great interest to the readers of Clinical Medicine \& Research. Despite a few glitches, healthcare providers are encouraged to take advantage of this helpful site. It is anticipated that as the audience grows and time goes by, Clinical Correlations will continue to mature into a quintessential electronic resource for general internal medicine practitioners and subspecialty providers.

\section{References}

1. Schmidt G. To blog or not to blog? Nat Geosci 2008;1:208.

2. Dearstyne BW. Blogs: the new information revolution? Information Management Journal 2005;39:38-44.

\section{Author Affiliations}

David M. Aronoff, MD*; Barbara A. Bartkowiak, MLIS, MST†

*Division of Infectious Diseases, Department of Internal Medicine, and Program in Biomedical Sciences, University of Michigan, Ann Arbor, Michigan, USA

†George E. Magnin Medical Library, Marshfield Clinic, Marshfield, Wisconsin, USA 